\title{
Sympathoinhibitory effect of statins in chronic heart failure
}

\author{
Marc E. Gomes · Jacques W. M. Lenders • \\ Louise Bellersen - Freek W. A. Verheugt • \\ Paul Smits • Cees J. Tack
}

Received: 10 July 2009/Accepted: 19 October 2009/Published online: 4 December 2009

(C) The Author(s) 2009. This article is published with open access at Springerlink.com

\begin{abstract}
Objectives Increased (central) sympathetic activity is a key feature of heart failure and associated with worse prognosis. Animal studies suggest that statin therapy can reduce central sympathetic outflow. This study assessed statin effects on (central) sympathetic activity in human chronic heart failure (CHF) patients.

Methods Sympathetic activity was measured in eight patients with CHF patients during 8 weeks after discontinuation and 4 weeks after restart of statin therapy by microneurography for direct muscle sympathetic nerve recording (MSNA) and measurement of arterial plasma norepinephrine concentrations.
\end{abstract}

M. E. Gomes · L. Bellersen · F. W. A. Verheugt

Department of Cardiology,

University Medical Center Nijmegen,

Nijmegen, The Netherlands

e-mail: mergomes@hotmail.com

J. W. M. Lenders · P. Smits

Department of Internal Medicine,

University Medical Center Nijmegen,

Nijmegen, The Netherlands

P. Smits

Department of Pharmacology Toxicololgy,

University Medical Center Nijmegen,

Nijmegen, The Netherlands

J. W. M. Lenders

Department of Medicine III, Carl Gustav Carus University

Medical Center, Dresden, Germany

C. J. Tack $(\square)$

Division of General Internal Medicine, University Hospital, Geert Grooteplein Zuid 8, PO Box 9101, 6500 HB Nijmegen, The Netherlands

e-mail: c.tack@aig.umcn.nl
Results During discontinuation of statin therapy, MSNA was significantly increased ( $73 \pm 4$ vs. $56 \pm 5$ and $52 \pm 6$ bursts $/ 100$ beats, $p=0.01$ ). Burst frequency was significantly higher after statin discontinuation $(42 \pm 3$ burst $/ \mathrm{min}$ without statin vs. $32 \pm 3$ and $28 \pm 3$ burst/min during statin therapy, $p=0.004)$. Mean normalized burst amplitude and total normalized MSNA were significantly higher after statin discontinuation (mean normalized burst amplitude $0.36 \pm 0.04$ without statin vs. $0.29 \pm 0.04$ and $0.22 \pm 0.04$ during statin, $p<0.05$; total normalized MSNA $15.70 \pm 2.78$ without statin, vs. $9.28 \pm 1.41$ and $6.56 \pm 1.83$ during statin, $p=0.009$ ). Arterial plasma norepinephrine levels and blood pressure were unaffected. Interpretation Statin therapy inhibits central sympathetic outflow in CHF patients, as measured by MSNA.

Keywords Heart failure - Nervous system, sympathetic . Statin · Catecholamines · HRV

\section{Introduction}

A number of cardiovascular conditions like chronic heart failure (CHF) is characterized by an increased sympathetic tone, reflecting the functional severity of CHF [4]. On the long term, this sympathetic overactivity has been shown to be detrimental, leading to an increased myocardial cell death, arrhythmias, reduced survival and increased hospitalization and morbidity rates $[1,2,16,22]$. Nowadays, reduction of sympathoexcitation by specific treatment has become a cornerstone in modern treatment of these conditions. Beta-adrenergic receptor antagonists and angiotensin-converting enzyme (ACE) inhibitors have been shown to increase survival and reduce morbidity [3, 24]. However, although these specific strategies shield target 
organs from increased sympathetic tone, the increased sympathetic nerve traffic itself remains unaffected.

In an animal model of pacing-induced $\mathrm{CHF}$, studies have shown that 3-hydroxy-3-methylglutaryl coenzyme A (HMG-CoA) reductase inhibitors (statins) reduce the enhanced sympathetic activity. However, the exact mechanism of this action of statins has not yet been clarified [5].

The primary aim of the present study was to test the hypothesis that statin therapy can inhibit sympathetic nerve activity also in humans. In this proof-of-concept study, we investigated patients with $\mathrm{CHF}$ because CHF is accompanied by increased sympathetic activity. Since patients with CHF are usually treated by statins, we hypothesized that temporary discontinuation of their statin therapy would increase sympathetic activity and restart of statin therapy would reduce it again. If statins do reduce sympathetic nerve activity in patients with $\mathrm{CHF}$, this effect might provide an additional explanation for the beneficial effects of statins in these patients [8].

\section{Methods}

\section{Participants}

Eight patients diagnosed with $\mathrm{CHF}$ according to the modified Framingham Clinical Criteria, New York Heart Association (NYHA) II-IV, were included in our study [21]. Inclusion criteria were left ventricular ejection fraction (LVEF) $\leq 40 \%$ as assessed by $2 \mathrm{D}$ echocardiography, stable statin treatment for at least 3 months prior to the study and sinus rhythm. Exclusion criteria were necessity of short-term percutaneous coronary intervention, coronary artery bypass grafting or heart transplantation, chronic disease of the autonomic nervous system, diabetes mellitus, an obligatory reason for not interrupting statin therapy (e.g. recent myocardial or cerebral infarction), use of tricyclic antidepressant drugs or $\alpha$-adrenergic receptor antagonists (or $\beta$-adrenergic receptor antagonists with $\alpha$-adrenergic receptor antagonizing properties). The investigation conforms with the principles outlined in the Declaration of Helsinki. The institutional review committee approved the study and informed consent was obtained from all patients before participation in the study.

\section{Study protocol}

Sympathetic activity was assessed using different approaches: direct measurement of muscle sympathetic nerve activity (MSNA) by microneurography and measurement of arterial plasma catecholamine concentrations. Effects on blood pressure, heart rate and cholesterol levels were recorded. Measurements were performed at 3 different time points: during statin therapy (visit 1), 8 weeks after discontinuation (visit 2) and 4 weeks after restart of (original) statin therapy (visit 3). Experiments were conducted at 8:00 a.m. after an overnight (10-h) fast with the patient in supine position in a quiet temperature-controlled room $\left(23-24^{\circ} \mathrm{C}\right)$. Subjects had to abstain from caffeine, tea, alcohol, chocolates and smoking for at least $12 \mathrm{~h}$ prior to the test procedure. All experiments were carried out after voiding to prevent reflex sympathoexcitation. A catheter (Angiocath: 20gauge, $48 \mathrm{~mm}$, Becton-Dickinson and Co., Sandy, Utah) was inserted under local anesthesia (lidocaine 2\%) into the brachial artery to obtain arterial blood samples and to have blood pressure measurements. After complete instrumentation, 30 min of rest were included to return to stable baseline conditions before data collection. Hereafter, arterial blood samples were drawn and microneurography was performed.

\section{Sympathetic nerve traffic (microneurography)}

The peroneal nerve was localized at the site of the fibula head using transdermal electrical impulses $(40-60 \mathrm{mV}$, $0.2 \mathrm{~ms}, 1 \mathrm{~Hz}$ ) by a small electrode. After localization, a sterile Tungsten microelectrode was inserted manually through the skin in the underlying peroneal nerve posterior to the fibular head. Electrode position within a muscle nerve fascicle was identified by evoking muscle twitches by electrical stimuli $(1-5 \mathrm{mV}, 0.2 \mathrm{~ms}, 1 \mathrm{~Hz})$ and through provoking afferent, mechanoreceptive activity by the stretching of the appropriate muscle. Then, minor adjustments of the electrode position were made to optimize the characteristic pattern of multi unit MSNA. From the original signal, multi-unit MSNA (m-MSNA) bursts were obtained as the average voltage after filtering (bandwidth of 700-2,000 Hz) and integrating (time constant $0.1 \mathrm{~s}$ ). The frequency and amplitude of MSNA bursts were obtained from bursts with a signal-to-noise ratio of $\geq 3$ [15]. Bursts were identified using an automated computer program with prefixed settings to exclude observer bias. A preset triangle with a base of $0.8 \mathrm{~s}$, correlated to the known delay between R-peak in the electrocardiogram (ECG) and the postganglionic burst, was used to scan the MSNA signal and identify sympathetic bursts. Hereafter, the maximal and average amplitude were calculated and potential bursts were correlated to a percentage of the maximal amplitude. The computer-derived measures hereafter were visually confirmed by an investigator so that noise artefacts were not being counted and if so, noise artefacts were removed. From the MSNA neurogram the following descriptors were used in the analysis (1) burst frequency (burst number/min), (2) burst incidence (burst number/100 heart beats), (3) mean normalized burst amplitude (burst amplitude normalized to the maximal burst in the recording) and (4) total normalized MSNA (sum of normalized burst amplitudes per min) [11]. 
Microneurography recordings were performed under baseline conditions (patient in supine position), which has a high intra-individual reproducibility [10]. Measurements were performed between 9:30 and 11:30, to prevent confounding by circadian variation [9].

Arterial plasma catecholamine levels

After a period of 20 min rest, plasma catecholamine concentrations were measured using high-performance liquid pressure analysis and fluorometric detection [26]. Within and between-run coefficients of variance (cv's) for plasma epinephrine (E) were 4.1 and $8.1 \%$ level of $0.166 \mathrm{nmol} / 1$ and for norepinephrine (NE) 4.1 and $6.1 \%$ at a level of $1.76 \mathrm{nmol} / \mathrm{l}$, respectively. Analytical detection limits were 0.003 and $0.002 \mathrm{nmol} / \mathrm{l}$ for $\mathrm{E}$ and NE, respectively.

Catecholamines were collected in ice-chilled $10 \mathrm{ml}$ Vacutainer tubes (Becton-Dickenson Co., Franklin Lakes, NJ, USA) containing $0.2 \mathrm{ml}$ of a solution of EGTA $(0.25 \mathrm{~mol} / \mathrm{L})$ and gluthatione $(0.20 \mathrm{~mol} / \mathrm{L})$.

Heart rate and blood pressure

Heart rate was measured continuously for a period of 10 min using a cardiotachometer triggered by the R-wave of an ECG lead. Simultaneously, intra-arterial systolic and diastolic blood pressure was measured using a standard transducer connected to the intra-arterial line. For further analysis, an average value for the 10-min interval was calculated for each parameter.

\section{Blood sampling}

Arterial blood samples were drawn 40 min after instrumentation. Fasting total cholesterol, low-density lipoprotein (LDL), high-density lipoprotein (HDL) and triglyceride levels were measured at each visit to assess the effect of statin therapy.

\section{Statistical analysis}

Data are expressed as mean \pm SEM unless indicated otherwise. Prior to the study, sample size needed to find a difference in sympathetic nervous system activity of $20 \%$ with a power of $80 \%$ was calculated to be $n=8$. Two-way ANOVA with the factors patient and visit was used to test the contrast for the difference between the visit during the withdrawal period and the mean of the visits during statin treatment. The distribution of some variables was skewed (heterogeneity) and these were $\log$ transformed which resulted in approximately Gaussian distributions and removed heterogeneity. A two-tailed $p$ value of $<0.05$ was considered to be statistically significant.
Table 1 Baseline echocardiographic data

\begin{tabular}{lc}
\hline Parameter & Value $( \pm$ SD) \\
\hline EF (\%) & $30 \pm 9$ \\
LVIDd (mm) & $61 \pm 9$ \\
RVIDd (mm) & $32 \pm 6$ \\
LVESV (ml) & $141 \pm 47$ \\
LVEDV (ml) & $188 \pm 55$ \\
E/A & $1.8 \pm 1.3$ \\
S/D & $1.1 \pm 0.6$ \\
\hline
\end{tabular}

$E F$ ejection fraction, $L V I D d$ diastolic left ventricle internal diameter, $R V I D d$ diastolic right ventricle internal diameter, $L V E S V$ left ventricle end-systolic volume, $L V E D V$ left ventricle end-diastolic volume, $E / A$ mitral valve E/A ratio, $S / D$ pulmonary vein $S / D$ ratio

\section{Results}

All eight male patients completed the study: mean NYHA class $( \pm \mathrm{SD})$ was $2.6 \pm 0.9$, age $55 \pm 8$ years and body mass index $31.4 \pm 6.6 \mathrm{~kg} / \mathrm{m}^{2}$. All patients suffered from residual myocardial ischemia after a previous myocardial infarction. Mean LVEF amounted to $30 \pm 9 \%$ (Table 1). Seven patients used simvastatin ( $40 \mathrm{mg}$ daily), one atorvastatin (40 mg daily). All patients used a $\beta$-blocker (5 patients atenolol, dosage ranging from 50 to $100 \mathrm{mg}$ daily; 2 patients metoprolol (25-200 $\mathrm{mg}$ ); and one patient bisoprolol (5 mg daily), all patients were on aspirin and an ACE-inhibitor (enalapril $n=1$, $\operatorname{losartan} n=2$, perindopril $n=3$, candasartan $n=1$, quinapril $n=1$ ). Seven patients used a diuretic (furosemide $n=2$, bumethanide $n=1$, spironolactone $n=4$ ) and one patient used a nitrate. These baseline medications remained unchanged throughout the course of the study in all patients.

\section{Sympathetic nerve traffic (microneurography)}

After 8 weeks of discontinuation of statins, MSNA was increased to $73 \pm 4$ bursts $/ 100$ beats $(p=0.01)$, while levels during statin were $56 \pm 5$ and $52 \pm 6$ bursts/100 beats (Fig. 1). Burst frequency was significantly higher after statin discontinuation (42 \pm 3 burst/min without statin vs. $32 \pm 3$ and $28 \pm 3$ burst/min during statin therapy, $p=0.004$ ). Mean normalized burst amplitude and total normalized MSNA were significantly higher after statin discontinuation (mean normalized burst amplitude $0.36 \pm$ 0.04 without statin vs. $0.29 \pm 0.04$ and $0.22 \pm 0.04$ during statin, $p<0.05$; total normalized MSNA $15.70 \pm 2.78$ without statin, vs. $9.28 \pm 1.41$ and $6.56 \pm 1.8$ during statin, $p=0.009$ ). In one out of the eight patients, we were unable to obtain qualitatively sufficient microneurography results to include in the analysis. No difference in MSNA responses to statins were found between patients with or without diuretics (or nitrate). 


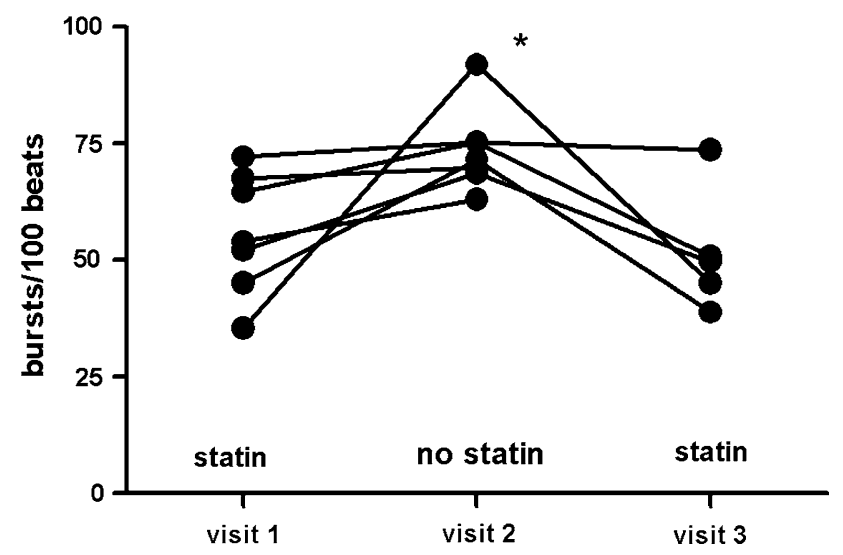

Fig. 1 Muscle sympathetic nerve activity (MSNA) in the individual CHF patients in bursts/100 heart beats at visit 1 (during statin therapy, $n=7$ ), visit 2 (8 weeks after statin discontinuation, $n=7$ ) and visit 3 (4 weeks after statin restart, $n=6$ ). MSNA was significantly higher after statin discontinuation. $* P=0.01$

Arterial plasma catecholamine levels and hemodynamics

Discontinuation of statin therapy did not affect arterial plasma NE and E levels (Table 2). Short-term statin discontinuation had no significant effect on systolic or diastolic blood pressure and heart rate (Table 1).

\section{Plasma cholesterol levels}

Discontinuation of statin therapy resulted in a significant increase in total cholesterol and LDL in all patients (Table 2), with no significant change in HDL cholesterol. These changes occurred consistently in all patients, consistent with good compliance to the study protocol.

\section{Discussion}

The present study provides evidence that statin therapy is able to suppress MSNA in CHF patients. During statin withdrawal, MSNA increased significantly. Short-term withdrawal of statins had no effect on arterial plasma catecholamine levels and blood pressure.

Our findings are in accordance with earlier data obtained in animal studies $[18,19]$. In normolipidemic rabbits with pacing-induced CHF, incremental doses of simvastatin reduced the increased renal sympathetic nervous system activity (RSNA) by nearly $50 \%$ compared to untreated animals. Gao et al. [5] showed a similar decrease in RSNA by statins in rabbits with pacing-induced CHF. In our study, MSNA increased by approximately $30 \%$ after statin discontinuation. When compared with the animal studies, this at a four to eightfold lower mean dosage of statin as calculated $\mathrm{kg}^{-1}$ body weight $\times \mathrm{day}^{-1}$. A recent study in patients with coronary artery disease also showed that atorvastatin shifted sympathovagal balance toward decreased sympathetic dominance [23]. In contrast, two prior studies in CHF patients failed to show an effect of $20 \mathrm{mg}$ simvastatin or $10 \mathrm{mg}$ atorvastatin on HRV, but this might be explained by the relative low-dose of statins used in these studies $[6,25]$. Our patients used twice the dosage of statins and since previous studies demonstrated a doseeffect relationship between statins and sympathetic activity, it is possible that the effects of statins on MSNA are only apparent at higher statin doses.

To date, the underlying mechanism by which statins reduce sympathetic outflow has not been resolved. Most hypothesis are derived from animal experimental data. Statins seem to enhance central nitric oxide (NO) concentrations in the paraventricular region, and down regulate

Table 2 Statin effects

$B P$ blood pressure, $E$ arterial plasma epinephrine concentration, $H D L$ high density lipoprotein, $L D L$ low density lipoprotein, $M A P$ mean arterial pressure, MSNA muscle nerve sympathetic activity, $N E$ arterial plasma norepinephrine concentration

$* P<0.05$ versus statin

\begin{tabular}{lccc}
\hline & Visit 1 (statin) & Visit 2 (no statin) & Visit 3 (statin) \\
\hline MSNA (bursts/100 beats) & $56 \pm 5$ & $73 \pm 4^{*}$ & $52 \pm 6$ \\
MSNA (burst/min) & $32 \pm 3$ & $42 \pm 3^{*}$ & $28 \pm 3$ \\
MSNA (mean normalized amplitude) & $0.29 \pm 0.04$ & $0.36 \pm 0.04^{*}$ & $0.22 \pm 0.04$ \\
MSNA (normalized total MSNA) & $9.28 \pm 1.41$ & $15.70 \pm 2.78^{*}$ & $6.56 \pm 1.83$ \\
NE (pg/ml) & $323 \pm 47$ & $286 \pm 39$ & $275 \pm 47$ \\
E (pg/ml) & $40 \pm 7$ & $29 \pm 4$ & $31 \pm 4$ \\
Systolic BP (mmHg) & $116 \pm 5$ & $118 \pm 6$ & $117 \pm 9$ \\
Diastolic BP (mmHg) & $61 \pm 3$ & $65 \pm 3$ & $60 \pm 3$ \\
MAP (mmHg) & $83 \pm 3$ & $85 \pm 4$ & $82 \pm 5$ \\
Heart rate (bpm) & $59 \pm 2$ & $60 \pm 3$ & $57 \pm 3$ \\
Total plasma cholesterol (mmol/L) & $3.4 \pm 0.4$ & $5.5 \pm 0.5^{*}$ & $3.5 \pm 0.4$ \\
LDL (mmol/L) & $1.8 \pm 0.4$ & $3.5 \pm 0.4^{*}$ & $1.7 \pm 0.2$ \\
HDL (mmol/L) & $1.0 \pm 0.1$ & $1.0 \pm 0.1$ & $0.9 \pm 0.1$ \\
Triglycerides (mmol/L) & $1.4 \pm 0.2$ & $2.5 \pm 0.4$ & $2.4 \pm 1.2$ \\
\hline
\end{tabular}


Angiotensin Type-1 receptor expression and NAD(P)H formation in the rostral ventrolateral medulla (RVLM) [5, 12]. Increases in central Ang II concentrations as seen in $\mathrm{CHF}$, stimulate and upregulate the $\mathrm{AT}_{1}$-receptor as well as NAD(P)H-oxidase expression [5]. Stimulating the $\mathrm{AT}_{1}$-receptor in turn stimulates $\mathrm{NAD}(\mathrm{P}) \mathrm{H}$-oxidase, resulting in increased reactive oxygen species (ROS) formation [5]. ROS increases sympathetic outflow in a number of ways. First, increases in ROS concentrations seem to reduce cerebral NO, a sympathoinhibitory substance [14]. Formed $\mathrm{NO}$ is immediately converted to peroxynitrite at high levels of intracerebral superoxide generation. Because $\mathrm{NO}$ is a well-known sympathoinhibitory substance, a reduction in bioavailability of $\mathrm{NO}$, would predispose local neurons in the hypothalamus and medulla to become more excitable and thereby generate an enhanced sympathetic outflow. Superoxide anion has also been shown to modulate calcium-channel function in the central nervous system [28]. This mechanism may contribute to neuronal excitability. Additionally, NO itself has also demonstrated to have a regulatory role on sympathetic outflow at a central level [17]. Although it is not exactly known how NO modulates sympathetic outflow, several scenarios have been suggested. Of these, the most important is the suggestion that NO production by neuronal nitric oxide synthase (nNOS) causes $\gamma$-amino butyric acid (GABA) release, that in turn produces a reduction in sympathetic outflow [27]. Abnormalities in the NO-mechanism may contribute to the high sympathetic output state of $\mathrm{CHF}$ and hypertension. Gao et al. [5] demonstrated in $\mathrm{CHF}$ rabbits that treatment with simvastatin down regulated $\mathrm{AT}_{1}$-receptor $\mathrm{mRNA}$ and protein expression and $\mathrm{NAD}(\mathrm{P}) \mathrm{H}$ oxidase components in the RVLM. If statins downregulate Angiotensin Type-1 receptor expression and $\mathrm{NAD}(\mathrm{P}) \mathrm{H}$ formation, as well as increase NO formation, this might offer a potential explanation how statins can reduce central sympathetic outflow. The current study, however, cannot differentiate between these mechanisms.

In our study, the changes in MSNA were not translated in a decrease in arterial plasma NE levels. How can this be explained? It might be possible that for an effect on plasma NE levels, higher doses of statins might be required. This is supported by a study in pacing-induced CHF rabbits [20]. Plasma NE concentrations were only reduced when administering statin doses up to $3 \mathrm{mg} / \mathrm{kg} / \mathrm{day}$, e.g. [18]. This dose level exceeds by far the normal human statin dose that was used in our study. In the low-dose group $(0.3 \mathrm{mg} / \mathrm{kg} /$ day $)$, plasma $\mathrm{NE}$ concentrations were not affected and this is in agreement with our study where the mean statin dosage was $0.4 \mathrm{mg} / \mathrm{kg} /$ day. Higher doses may be able to suppress plasma NE levels as is suggested by a recent study, demonstrating a significant reduction in plasma NE concentrations after administering atorvastatin in a dose of $80 \mathrm{mg}$ to patients with coronary artery disease [23]. Thus, the lack of change in plasma arterial NE levels is in accordance with animal studies and may be explained by the relative low statin dose. An alternative explanation might be that the plasma NE level is the net result of plasma NE spillover and NE clearance. A decrease in neuronal NE release by the decrease in MSNA might be translated into a decreased plasma NE spillover. However, a concomitant increase in plasma NE clearance might mask any effect of plasma NE spillover on plasma NE levels, leaving the latter unaltered. Finally, it should be noted that MSNA reflects only the sympathetic outflow to the muscle compartment while plasma NE levels represent merely global total body sympathetic nerve activity. So, even if statins reduce sympathetic nerve traffic to the muscle compartment, any contrasting change in sympathetic nerve traffic to another compartment might result in an unaltered plasma NE level.

In line with the decrease in sympathetic nerve traffic, one would expect a decrease in blood pressure or heart rate but this was not observed. This is probably due to the fact that effects on blood pressure and heart rate are also determined by other compensatory regulatory mechanisms like the renin-angiotensin-aldosterone system [22]. Although non-compliance to the drug might also explain this effect, the decrease in plasma lipids in all patients argues against this possibility. Alternatively, the sympathoinhibitory effect of this dose statins is not large enough to be translated into a decrease in blood pressure or heart rate. Despite a reduction in plasma NE levels, statin doses as high as $3 \mathrm{mg} / \mathrm{kg} /$ day did not affect hemodynamics in $\mathrm{CHF}$ rabbits [18].

One may question whether the sympathoinhibitory effect of statin therapy would have any additional beneficial effect on top of betablocking therapy in patients with CHF. Beta-adrenoreceptor antagonists protect the cardiovascular system from sympathetic stimulation by blocking the $\beta$-adrenoceptors. However, vascular postsynaptic $\alpha$-adrenoreceptors are left unblocked and are therefore still operational in determining peripheral vascular resistance. The uninterrupted stimulating effect of increased sympathetic nerve traffic on these $\alpha$-adrenoreceptors might be detrimental for the cardiovascular system [20]. If statins indeed reduce sympathetic outflow, as suggested by our study, they may offer an additional pathway to counteract the increased sympathetic tone, thereby reducing its detrimental effects [20]. Two recent large clinical trials (GISSIHF and CORONA) could not establish a beneficial effect of statins on clinical outcome in CHF patients [7, 13]. This suggests that the reduction of sympathetic nerve traffic by statins is possibly insufficient to be translated into beneficial effects on long-term cardiovascular morbidity and mortality. An alternative explanation is that $\mathrm{CHF}$ in these 
large studies was caused by different underlying cardiac disorders or that different statins and/or doses that were used in these studies.

In conclusion, the results of this study suggest that statins may have a sympathoinhibitory in patients with CHF. If this effect is confirmed by larger scale studies, statins might play an additional protective role in cardiovascular patients apart from their effects on lipids, since an increased sympathetic tone may be detrimental on the long term.

Acknowledgments This work was supported by ZonMw of the Netherlands Organization for Health Research and Development (Marc E. Gomes). Cees J. Tack is a recipient of a Clinical Research Fellowship and is supported by National Institute of Diabetes and Digestive and Kidney Disease-DK 069881.

Conflict of interest statement No conflicts of interest for all authors.

Open Access This article is distributed under the terms of the Creative Commons Attribution Noncommercial License which permits any noncommercial use, distribution, and reproduction in any medium, provided the original author(s) and source are credited.

\section{References}

1. Benedict CR, Shelton B, Johnstone DE et al (1996) Prognostic significance of plasma norepinephrine in patients with asymptomatic left ventricular dysfunction. SOLVD Investigators. Circulation 94:690-697

2. Cohn JN, Levine TB, Olivari MT et al (1984) Plasma norepinephrine as a guide to prognosis in patients with chronic congestive heart failure. N Engl J Med 311:819-823

3. Di Lenarda A, Sabbadini G, Moretti M, Sinagra G (2004) Longterm oral carvedilol in chronic heart failure. Expert Opin Pharmacother 5:1359-1372

4. Francis GS, Goldsmith SR, Levine TB, Olivari MT, Cohn JN (1984) The neurohumoral axis in congestive heart failure. Ann Intern Med 101:370-377

5. Gao L, Wang W, Li YL et al (2005) Simvastatin therapy normalizes sympathetic neural control in experimental heart failure: roles of angiotensin II type 1 receptors and $\mathrm{NAD}(\mathrm{P}) \mathrm{H}$ oxidase. Circulation 112:1763-1770

6. Gentlesk PJ, Wiley T, Taylor AJ (2005) A prospective evaluation of the effect of simvastatin on heart rate variability in nonischemic cardiomyopathy. Am Heart J 150:478-483

7. Gissi-HF I, Tavazzi L, Maggioni AP et al (2008) Effect of rosuvastatin in patients with chronic heart failure (the GISSI-HF trial): a randomised, double-blind, placebo-controlled trial. Lancet 372:1231-1239

8. Go AS, Lee WY, Yang J, Lo JC, Gurwitz JH (2006) Statin therapy and risks for death and hospitalization in chronic heart failure. JAMA 296:2105-2111

9. Grassi G, Esler M (1999) How to assess sympathetic activity in humans. J Hypertens 17:719-734
10. Grassi G, Seravalle G, Cattaneo BM et al (1995) Sympathetic activation and loss of reflex sympathetic control in mild congestive heart failure. Circulation 92:3206-3211

11. Kimmerly DS, O'Leary DD, Shoemaker JK (2004) Test-retest repeatability of muscle sympathetic nerve activity: influence of data analysis and head-up tilt. Auton Neurosci 114:61-71

12. Kishi T, Hirooka Y, Mukai Y, Shimokawa H, Takeshita A (2003) Atorvastatin causes depressor and sympatho-inhibitory effects with upregulation of nitric oxide synthases in stroke-prone spontaneously hypertensive rats. J Hypertens 21:379-386

13. Kjekshus J, Apetrei E, Barrios V et al (2007) Rosuvastatin in older patients with systolic heart failure. N Engl J Med 357:22482261

14. Lassegue B, Griendling KK (2004) Reactive oxygen species in hypertension: an update. Am J Hypertens 17:852-860

15. Mano T, Iwase S, Toma S (2006) Microneurography as a tool in clinical neurophysiology to investigate peripheral neural traffic in humans. Clin Neurophysiol 117:2357-2384

16. Packer M, Lee WH, Kessler PD, Gottlieb SS, Bernstein JL, Kukin ML (1987) Role of neurohormonal mechanisms in determining survival in patients with severe chronic heart failure. Circulation 75:IV80-IV92

17. Patel KP, Li YF, Hirooka Y (2001) Role of nitric oxide in central sympathetic outflow. Exp Biol Med 226:814-824

18. Pliquett RU, Cornish KG, Peuler JD, Zucker IH (2003) Simvastatin normalizes autonomic neural control in experimental heart failure. Circulation 107:2493-2498

19. Pliquett RU, Cornish KG, Zucker IH (2003) Statin therapy restores sympathovagal balance in experimental heart failure. J Appl Physiol 95:700-704

20. Reaven GM, Lithell H, Landsberg L (1996) Hypertension and associated metabolic abnormalities - the role of insulin resistance and the sympathoadrenal system. N Engl J Med 334:374-381

21. Senni M, Tribouilloy CM, Rodeheffer RJ et al (1998) Congestive heart failure in the community: a study of all incident cases in Olmsted County, Minnesota, in 1991. Circulation 98:2282-2289

22. Swedberg K, Eneroth P, Kjekshus J, Wilhelmsen L (1990) Hormones regulating cardiovascular function in patients with severe congestive-heart-failure and their relation to mortality. Circulation 82:1730-1736

23. Szramka M, Harriss L, Ninnio D et al (2007) The effect of rapid lipid lowering with atorvastatin on autonomic parameters in patients with coronary artery disease. Int J Cardiol 117(2):287-291

24. The SOLVD Investigators (1991) Effect of enalapril on survival in patients with reduced left ventricular ejection fractions and congestive heart failure. N Engl J Med 325:293-302

25. Vrtovec B, Okrajsek R, Golicnik A, Ferjan M, Starc V, Radovancevic B (2005) Atorvastatin therapy increases heart rate variability, decreases QT variability, and shortens QTc interval duration in patients with advanced chronic heart failure. J Card Fail 11:684-690

26. Willemsen JJ, Ross HA, Jacobs MC et al (1995) Highly sensitive and specific HPLC with fluorometric detection for determination of plasma epinephrine and norepinephrine applied to kinetic studies in humans. Clin Chem 41:1455-1460

27. Zanzinger J, Czachurski J, Seller H (1997) Neuronal nitric oxide reduces sympathetic excitability by modulation of central glutamate effects in pigs. Circ Res 80:565-571

28. Zimmerman MC, Sharma RV, Davisson RL (2005) Superoxide mediates angiotensin II-induced influx of extracellular calcium in neural cells. Hypertension 45:717-723 